\title{
Genomic Analysis of a Pathogenic Bacterium, Paeniclostridium sordellii CBA7122 Containing the Highest Number of rRNA Operons, Isolated from a Human Stool Sample
}

\author{
Joon Yong Kim ${ }^{1}$, Yeon Bee Kim ${ }^{1}$, Hye Seon Song ${ }^{1}$, Won-Hyong Chung ${ }^{2}$, Changsu Lee ${ }^{1}$, \\ Seung Woo Ahn ${ }^{1}$, Se Hee Lee ${ }^{1}$, Min Young Jung ${ }^{1}$, Tae-Woon Kim ${ }^{1}$, Young-Do Nam ${ }^{2,3 *}$ and \\ Seong Woon Roh ${ }^{1 *}$ \\ ${ }^{1}$ Microbiology and Functionality Research Group, World Institute of Kimchi, Gwangju, South Korea, ${ }^{2}$ Research Group of Gut \\ Microbiome, Korea Food Research Institute, Sungnam, South Korea, ${ }^{3}$ Department of Food Biotechnology, University of \\ Science and Technology, Daejeon, South Korea
}

\section{OPEN ACCESS}

Edited by:

Annalisa Bruno,

Università degli Studi "G. d'Annunzio"

Chieti - Pescara, Italy

Reviewed by:

Bradley Stevenson,

University of Oklahoma, United States

Annalisa Trenti,

Università degli Studi di Padova, Italy

*Correspondence:

Seong Woon Roh

swroh@wikim.re.kr

Young-Do Nam

youngdo98@kfri.re.kr

Specialty section: This article was submitted to Inflammation Pharmacology,

a section of the journal

Frontiers in Pharmacology

Received: 05 September 2017 Accepted: 03 November 2017 Published: 15 November 2017

Citation:

Kim JY, Kim YB, Song HS, Chung W-H, Lee C, Ahn SW, Lee SH,

Jung MY, Kim T-W, Nam Y-D and Roh SW (2017) Genomic Analysis of a

Pathogenic Bacterium,

Paeniclostridium sordellii CBA7122 Containing the Highest Number of rRNA Operons, Isolated from a Human Stool Sample.

Front. Pharmacol. 8:840.

doi: 10.3389/fphar.2017.00840
Keywords: Paeniclostridium sordellii, pathogen, genome sequence, rRNA operon, virulence factor

\section{INTRODUCTION}

Paeniclostridium sordellii was first isolated by Alfredo Sordelli in 1922 under the proposed name Bacillus oedematis, and was then renamed Bacillus sordellii in 1927 (Hall and Scott, 1927). Two years later, it was classified as Clostridium sordellii (Hall et al., 1929). Recently, this bacterium was reclassified as a species of the genus Paeniclostridium, named P. sordellii comb. nov. (Sasi Jyothsna et al., 2016). P. sordellii is an anaerobic, Gram-stain-positive, spore-forming rod bacterium with flagella. Most strains are non-pathogenic, but some strains have been associated with severe infections of humans and animals. In humans, $P$. sordellii is mainly associated with trauma, toxic shock, soft tissue skin infections, and gynecologic infections. Despite the serious consequences of infection with $P$. sordellii, treatment is difficult because of the rapid progression from recognition of the first symptoms to death (Aldape et al., 2006).

In this study, we performed whole-genome sequencing and genomic analysis of strain CBA7122 belonging to $P$. sordellii, which was isolated from the stool sample of an 85-year-old healthy female residing in the Republic of Korea. This genomic information of $P$. sordellii CBA7122 should motivate further research on related strains, which may provide new insight into the pathogenesis of $P$. sordellii toward development of new strategies for the control, prevention, and treatment of life-threatening infections.

\section{MATERIALS AND METHODS}

\section{Strain Isolation, Culture, and DNA Extraction}

Strain CBA7122 was isolated from the stool sample of an 85 -year-old healthy female by the standard dilution plating technique on modified Eggerth-Gagnon agar medium (containing per liter of distilled water: $10 \mathrm{~g}$ peptone, $4 \mathrm{~g} \mathrm{Na}_{2} \mathrm{HPO}_{4}, 2 \mathrm{~g}$ porcine gastric mucin, $50 \mathrm{ml}$ sheep blood, $15 \mathrm{~g}$ agar) at $37^{\circ} \mathrm{C}$ for $24 \mathrm{~h}$ in an anaerobic chamber (Coy Laboratory Products) with an atmosphere of $\mathrm{N}_{2} / \mathrm{CO}_{2} / \mathrm{H}_{2}$ (90:5:5, by volume). Routine cultivation of strain CBA7122 was performed under the same conditions. Genomic DNA of strain CBA7122 was extracted using the QIAamp DNA extraction kit (Qiagen, USA) and QuickGene DNA tissue kit S (Kurabo, Japan), and quantified using the Quant-iT PicoGreen dsDNA Assay kit (Invitrogen, USA). The condition of extracted DNA was assessed by agarose gel electrophoresis on a $1 \%$ agarose gel. 


\section{Genome Sequencing, Assembly, and Annotation}

The genome sequencing of strain CBA7122 was performed using a PacBio RS II sequencing platform as described previously (Kim et al., 2016). The library based on the genomic DNA of strain CBA7122 was constructed according to the manufacturer's instructions, and sequenced using a Pacific Biosciences RS II instrument. The 150,292 generated reads were filtered and assembled using the HGAP 2 protocol with default parameters in SMRT Analysis version 2.3. Gene prediction and the basic annotation for the assembled genome of strain CBA7122 were performed using the NCBI Prokaryotic Genome Annotation Pipeline (PGAP) (Tatusova et al., 2016). In brief, 16S rRNAs and $23 \mathrm{~S}$ rRNAs were predicted using BLASTn, and 5S rRNAs and small ncRNAs were predicted using cmsearch 1.1.1. tRNAscan-SE was used to predict tRNA gene sequences. Coding sequences (CDSs) were detected using GenMarkS+. Functional gene annotations of the genome of strain CBA7122 were performed against various databases, including the catalytic families (CatFam) (Yu et al., 2009), Clusters of Orthologous Groups (COG) (Tatusov et al., 2000), NCBI reference sequence (RefSeq) (O'Leary et al., 2016), and SEED (Overbeek et al., 2014) databases. CRISPRs were confirmed using CRISPRFinder (Grissa et al., 2007). Prophage analysis was performed by PHAST (Arndt et al., 2016).

\section{Comparative Genomic Analysis}

To find unique features of the genome of strain CBA7122, the genomes of the following P. sordellii and Paraclostridium bifermentans strains were selected to perform comparative genomic analysis using the NCBI genome database (http:// www.ncbi.nlm.nih.gov/genome/): $P$. sordellii strains ATCC 9714 ${ }^{\mathrm{T}}$ (GCA_000444075.1), VPI 9048 (GCA_000444095.1), and JGS6382 (GCA_000953555.1), and P. bifermentans ATCC $638^{\mathrm{T}}$ (GCA_000452245.1). Genome similarities between strain CBA7122 and the reference strains were determined using Orthologous Average Nucleotide Identity (OrthoANI) values, and used to reconstruct the phylogenetic tree using the Orthologous Average Nucleotide Identity Tool (Lee et al., 2016). For comparisons at the whole-genome level, the genomes of strain CBA7122 and related strains were aligned using the progressive MAUVE algorithm in the MAUVE multiple genome alignment software 2.4.0 (Darling et al., 2004). Pangenome analysis was performed by BIOiPLUG (Chunlab, Korea).

\section{Virulence Factor Identification}

To determine the virulence factors of strain CBA7122, Basic Local Alignment Search Tool (BLAST) was used with the core dataset containing information on genes associated with experimentally verified virulence factors in the virulence factor database (VFDB) (Chen et al., 2016), with the expected e-value 0.0001 .

\section{Ethics Statement}

The study protocol was approved by the institutional review board of the Theragen ETEX Bio Institute
(700062-20160804-JR-005-02). Before the current study, the purpose, experimental procedure, and benefits were fully explained to the participants. Oral consents were obtained from each volunteer and consent procedure was witnessed and documented on the research record.

\section{RESULTS}

\section{General Genomic Features of $\boldsymbol{P}$. sordellii CBA7122}

The genome of $P$. sordellii strain CBA7122 comprised 3 contigs and was 3,550,411 bp long. Based on NCBI PGAP, a total of 3,459 genes were predicted, including 17 16S-23S$5 S$ rRNA operons (51 rRNAs), 105 tRNAs, and 4 ncRNAs. The circular map of the genome is displayed in Figure 1, and detailed genome features of strain CBA7122 are listed in Table 1. Among the COG categories, 2,919 genes were categorized to transcription (230 genes); amino acid transport and metabolism (223); energy production and conversion (174); translation, ribosomal structure, and biogenesis (164); signal transduction mechanisms (156); cell wall/membrane/envelope biogenesis (155); carbohydrate transport and metabolism (153); inorganic ion transport and metabolism (143); replication, recombination, and repair (133); coenzyme transport and metabolism (93); nucleotide transport and metabolism (86); posttranslational modification, protein turnover, and chaperones (82); lipid transport and metabolism (45); cell motility (43); cell cycle control, cell division, and chromosome partitioning (33); secondary metabolites biosynthesis, transport, and catabolism (22); and function unknown (984). In the SEED database, a total of 1,470 genes were matched to the subsystem as follows: protein metabolism (264 genes); amino acids and derivatives (228); cofactors, vitamins, prosthetic groups, and pigments (206); carbohydrates (189); cell wall and capsule (169); DNA metabolism (110); nucleosides and nucleotides (105); RNA metabolism (104); virulence, disease, and defense (86); membrane transport (80); fatty acids, lipids, and isoprenoids (80); stress response (80); dormancy and sporulation (69); motility and chemotaxis (65); phosphorus metabolism (42); respiration (39); cell division and cell cycle (38); phages, prophages, transposable elements, and plasmids (28); regulation and cell signaling (25); sulfur metabolism (18); iron acquisition and metabolism (17); miscellaneous (14); potassium metabolism (11); nitrogen metabolism (9); and metabolism of aromatic compounds (1). According to CRISPR analysis, strain CBA7122 did not have known CRISPRs. Strain CBA7122 had two intact phage genomes (30,978 bp long and $41,432 \mathrm{bp}$ long), located in contig 2. Furthermore, 12,687 of the 16,833-bp-long contig 1 matched to incomplete phage genes.

\section{The Number of rRNA Gene Copies of P. sordellii CBA7122}

Interestingly, the genome of strain CBA7122 had 17 rRNA operons, which represented the highest number of rRNA operons known so far in the domain Bacteria. Of all P. sordellii genomes 

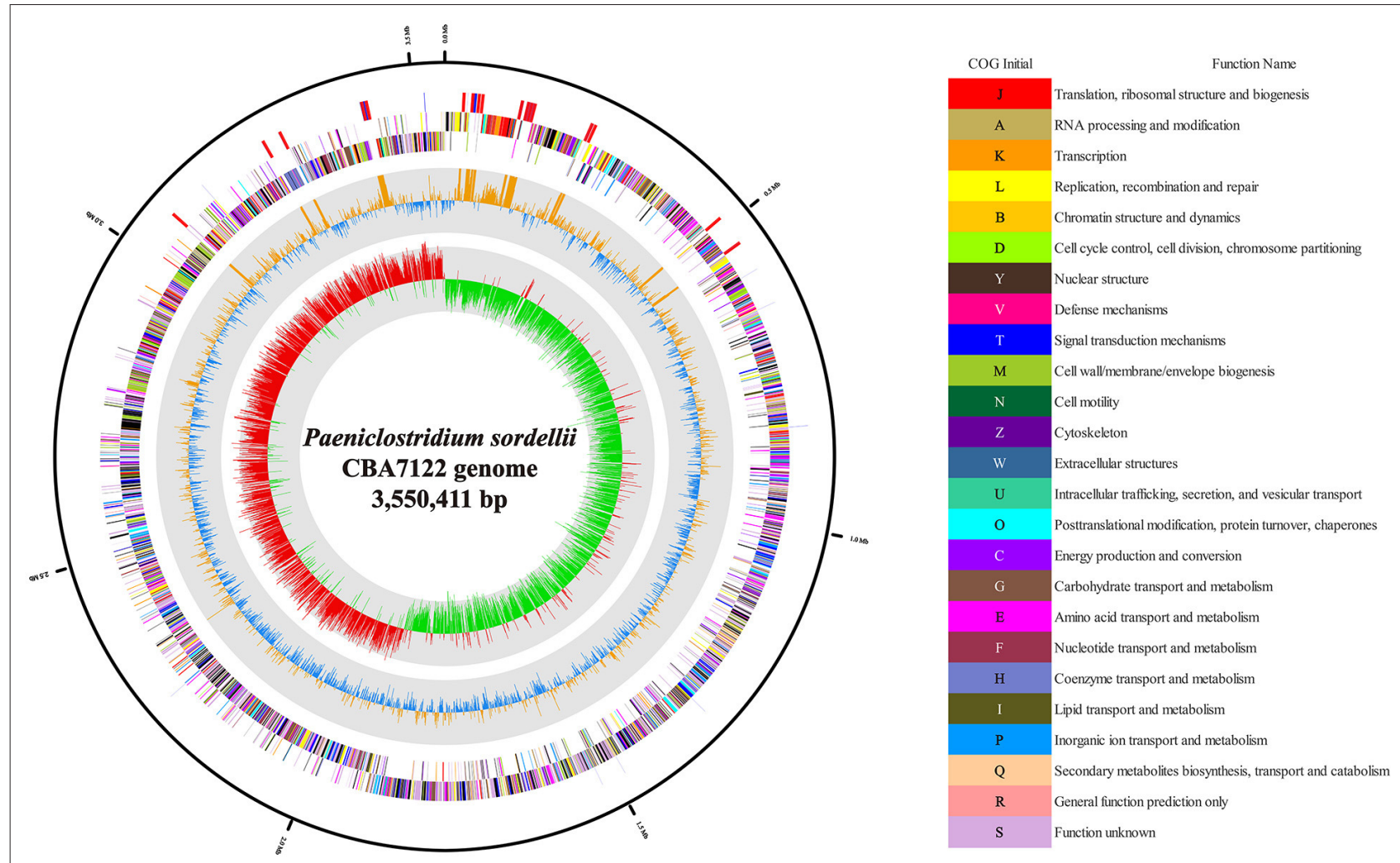

FIGURE 1 | Circular map of the Paeniclostridium sordellii CBA7122 genome. RNA genes (red, rRNA; blue, tRNA), forward and reverse strands (colored according to COG categories) are indicated from the outer fringe to the center. Inner circles show the GC content in yellow and blue and the GC skew is shown with red and green indicating positive and negative values, respectively. This genome map was generated by CLgenomics 1.52 (Chun Lab Inc.).

TABLE 1 | General genome features of Paeniclostridium sordellii CBA7122.

\begin{tabular}{ll}
\hline Item & Values \\
\hline Finishing quality & Draft \\
Sequencing platform & PacBio RS II \\
Assembler & PacBio SMRT Analysis 2.3.0 \\
Methods reads & 82,499 \\
Genome coverage & $298.06 \times$ \\
Assembly size (bp) & $3,550,411$ \\
N50 & $3,527,419$ \\
DNA G + C content (\%) & 27.8 \\
Total contigs & 3 \\
Total genes & 3,459 \\
Total CDSs & 3,299 \\
RNA genes & 160 \\
rRNAs & 17 rRNA operons (16S-23S-5S) \\
tRNAs & 105 \\
ncRNAs & 4 \\
\hline
\end{tabular}

listed in the NCBI genome database, strain CBA7122 had the highest $16 \mathrm{~S}$ and $23 \mathrm{~S}$ rRNA genes copies, whereas other genomes had lower $16 \mathrm{~S}$ (3.7 in average) and $23 \mathrm{~S}$ (5.5 in average) rRNA genes copies (Supplementary Figure S1). In the case of the number of $5 \mathrm{~S}$ rRNA genes, all genomes had an average of
13.7 gene copies, and strain CBA7122 had the second most number of gene copies. According to Roller et al. (2016), the number of rRNA operons is known to be associated with these two factors of reproduction, growth rate, and growth efficiency.

\section{Comparative Genomic Analysis}

Based on the OrthoANI values, strain CBA7122 had greatest sequence similarity with $P$. sordellii strain JGS6382 (98.74\%), followed by $P$. sordellii strains VPI 9048 (98.69\%) and ATCC $9714^{\mathrm{T}}(98.31 \%)$, and was most dissimilar to P. bifermentans ATCC $638^{\mathrm{T}}(81.14 \%)$. The phylogenetic tree based on orthoANI values supported that strain CBA7122 is closely related to $P$. sordellii (Supplementary Figure S2). Whole-genome comparison of strain CBA7122 with $P$. sordellii JGS6382, VPI 9048, and ATCC $9714^{\mathrm{T}}$, and P. bifermentans ATCC $638^{\mathrm{T}}$ revealed that most of locally collinear blocks (LCBs) are closely homologous within the species rather than genus (Supplementary Figure S3). In the pan-genome analysis, a total of 4,856 pan-genome orthologous groups (POGs) were obtained from the 17,352 CDSs of the 5 genomes. As shown in Supplementary Figure S4, the core genome comprises 2,481 POGs and the genome of strain CBA7122 contains only 145 POGs as singletons. Of the 48 genes in these 145 singleton POGs, 36 were annotated to the SEED 
subsystem, including cell wall and capsule (8 genes); amino acids and derivatives (5); DNA metabolism (5); motility and chemotaxis (4); phages, prophages, transposable elements, and plasmids (2); carbohydrates (1); miscellaneous (1); nucleosides and nucleotides (1); phosphorus metabolism (1); RNA metabolism (1); and virulence, disease, and defense subsystem (1). The others were annotated as hypothetical or uncharacterized proteins (Supplementary Table S1).

\section{Virulence Factors}

We identified several known virulence factors in the genome of strain CBA7122, including perfringolysin $\mathrm{O}\left(p f_{0} A\right)$, sialidase (nanH), thiol-activated cytolysin $(A L O)$, polysialic acid capsule biosynthesis protein $\mathrm{SiaC}$ (siaC), Hsp60, 60K heat shock protein HtpB $(h t p B)$, capsular polysaccharide synthesis enzyme Cap8D (cap8D), UDP-galactopyranose mutase (cpsI), UDP-glucose 6dehydrogenase (hasB), ATPase EscN (escN), glycosyl transferase CpsE (cpsE), Listeria adhesion protein Lap (lap), ATP-dependent protease $(c l p E)$, sigma 54-dependent response regulator $($ fleR/flrC), collagenase (colA), and UDP-galactopyranose mutase $(\mathrm{glf})$. However, there were no large clostridial cytotoxin (LCC) genes identified in the genome of strain CBA7122, whereas these genes were identified in strains ATCC $9714^{\mathrm{T}}$ and VPI 9048, which are known as the key factors of human infection leading to death.

Our data based on genomic analyses provides basic information of $P$. sordellii, which should serve as a useful reference for detailed studies focused on gaining a better understanding of the virulence factors in the genomes of these strains and their effects on human health. In addition, since strain CBA7122 contains a prophage genome, it is necessary to check whether the human infection

\section{REFERENCES}

Aldape, M. J., Bryant, A. E., and Stevens, D. L. (2006). Clostridium sordellii infection: epidemiology, clinical findings, and current perspectives on diagnosis and treatment. Clin. Infect. Dis. 43, 1436-1446. doi: 10.1086/508866

Arndt, D., Grant, J. R., Marcu, A., Sajed, T., Pon, A., Liang, Y., et al. (2016). PHASTER: a better, faster version of the PHAST phage search tool. Nucleic Acids Res. 44, W16-W21. doi: 10.1093/nar/gkw387

Chen, L., Zheng, D., Liu, B., Yang, J., and Jin, Q. (2016). VFDB 2016: hierarchical and refined dataset for big data analysis-10 years on. Nucleic Acids Res. 44, D694-D697. doi: 10.1093/nar/gkv1239

Darling, A. C., Mau, B., Blattner, F. R., and Perna, N. T. (2004). Mauve: multiple alignment of conserved genomic sequence with rearrangements. Genome Res. 14, 1394-1403. doi: 10.1101/gr.2289704

Grissa, I., Vergnaud, G., and Pourcel, C. (2007). CRISPRFinder: a web tool to identify clustered regularly interspaced short palindromic repeats. Nucleic Acids Res. 35, W52-W57. doi: 10.1093/nar/gkm360

Hall, I. C., Rymer, M. R., and Jungherr, E. (1929). Comparative Study of Bacillus sordellii (Hall and Scott) and Clostridium Oedematoides (Meleney, Humphreys and Carp). J. Infect. Dis. 45, 42-60. doi: 10.1093/infdis/45.1.42

Hall, I. C., and Scott, J. P. (1927). Bacillus Sordellii, a cause of malignant edema in man. J. Infect. Dis. 41, 329-355. doi: 10.1093/infdis/41.5.329

Kim, J. Y., Song, H. S., Kim, Y. B., Kwon, J., Choi, J. S., Cho, Y. J., et al. (2016). Genome sequence of a commensal bacterium, Enterococcus status will be changed through phage therapy in further studies.

\section{DATA ACCESS}

The genome sequence of Paeniclostridium sordellii CBA7122 were deposited in the DDBJ/ENA/GenBank under accession numbers BDJI01000001-BDJI01000003.

\section{AUTHOR CONTRIBUTIONS}

SWR and Y-DN designed and coordinated all the experiments. HSS performed cultivation, DNA extraction, and purification. JYK, YBK, SHL, and MYJ performed the sequence assembly, gene prediction, gene annotation, comparative genomic analysis, and wrote manuscript. W-HJ, CL, SWA, and T-WK checked and edited the manuscript. All authors have read and approved the manuscript.

\section{FUNDING}

This research was supported by grants from the World Institute of Kimchi (KE1702-2), funded by the Ministry of Science and ICT, Korea Food Research Institute (E0170602-01), and Basic Science Research Program through the National Research Foundation of Korea (NRF) (2015R1D1A1A09061039), Republic of Korea.

\section{SUPPLEMENTARY MATERIAL}

The Supplementary Material for this article can be found online at: https:/www.frontiersin.org/articles/10.3389/fphar. 2017.00840/full\#supplementary-material

faecalis CBA7120, isolated from a Korean fecal sample. Gut Pathog. 8:62. doi: 10.1186/s13099-016-0145-X

Lee, I., Kim, Y. O., Park, S. C., and Chun, J. (2016). OrthoANI: an improved algorithm and software for calculating average nucleotide identity. Int. J. Syst. Evol. Microbiol. 66, 1100-1103. doi: 10.1099/ijsem.0. 000760

O'Leary, N. A., Wright, M. W., Brister, J. R., Ciufo, S., Haddad, D., McVeigh, R., et al. (2016). Reference sequence (RefSeq) database at NCBI: current status, taxonomic expansion, and functional annotation. Nucleic Acids Res. 44, D733-D745. doi: 10.1093/nar/gkv1189

Overbeek, R., Olson, R., Pusch, G. D., Olsen, G. J., Davis, J. J., Disz, T., et al. (2014). The SEED and the Rapid Annotation of microbial genomes using Subsystems Technology (RAST). Nucleic Acids Res. 42, D206-D214. doi: $10.1093 /$ nar/gkt1226

Roller, B. R., Stoddard, S. F., and Schmidt, T. M. (2016). Exploiting rRNA operon copy number to investigate bacterial reproductive strategies. Nat. Microbiol. 1:16160. doi: 10.1038/nmicrobiol. 2016.160

Sasi Jyothsna, T. S., Tushar, L., Sasikala, C., and Ramana, C. V. (2016). Paraclostridium benzoelyticum gen. nov. sp. nov., isolated from marine sediment and reclassification of Clostridium bifermentans as Paraclostridium bifermentans comb. nov. Proposal of a new genus Paeniclostridium gen. nov. to accommodate Clostridium sordellii and Clostridium ghonii. Int. J. Syst. Evol. Microbiol. 66, 2459-2459. doi: 10.1099/ijsem.0.001144 
Tatusov, R. L., Galperin, M. Y., Natale, D. A., and Koonin, E. V. (2000). The COG database: a tool for genome-scale analysis of protein functions and evolution. Nucleic Acids Res. 28, 33-36. doi: 10.1093/nar/ 28.1 .33

Tatusova, T., DiCuccio, M., Badretdin, A., Chetvernin, V., Nawrocki, E. P., Zaslavsky, L., et al. (2016). NCBI prokaryotic genome annotation pipeline. Nucleic Acids Res. 44, 6614-6624. doi: 10.1093/nar/gkw569

Yu, C., Zavaljevski, N., Desai, V., and Reifman, J. (2009). Genomewide enzyme annotation with precision control: catalytic families (CatFam) databases. Proteins 74, 449-460. doi: 10.1002/prot. 22167
Conflict of Interest Statement: The authors declare that the research was conducted in the absence of any commercial or financial relationships that could be construed as a potential conflict of interest.

Copyright (C) 2017 Kim, Kim, Song, Chung, Lee, Ahn, Lee, Jung, Kim, Nam and Roh. This is an open-access article distributed under the terms of the Creative Commons Attribution License (CC BY). The use, distribution or reproduction in other forums is permitted, provided the original author(s) or licensor are credited and that the original publication in this journal is cited, in accordance with accepted academic practice. No use, distribution or reproduction is permitted which does not comply with these terms. 